Thomas Gloning

\title{
Textorganisation und Wortgebrauch im mittelniederdeutschen Gaerde der suntheit (1492) ${ }^{1}$
}

\section{Einleitung}

Die mittelalterlichen und frühneuzeitlichen Kräuterbücher und Simplizien- bzw. Drogenverzeichnisse sind eine wichtige Quelle für den medizinischen Sprachgebrauch des Mittelalters und der Frühen Neuzeit. Von der mhd. Macer-Übersetzung bis $\mathrm{zu}$ den monumentalen, vielhundertseitigen Kräuterbüchern des 16.-18. Jhs. verläuft eine stetige Tradition, die sich durch fortschreitende Anreicherung und ggf. auch Umbildung der Text- und Wissensbestände, durch die seit dem 16. Jh. verstärkt übliche Berücksichtigung von botanischen Informationen im engeren Sinne, durch Veränderungen in der Bildnutzung und durch eine Vielzahl von sprachlichen Veränderungen im Bereich des Wortgebrauchs, der syntaktischen Muster, der textuellen Organisation, der Schreibweisen usw. geprägt war. Manche dieser Entwicklungen, z.B. die schreibsprachlichen, gehören eher in den Bereich der allgemeinen Sprachentwicklung, andere, z.B. die Entwicklung und Standardisierung textueller Schemata, sind sehr viel enger an den Texttyp ,Pflanzenmonographie‘ als zentralen Baustein von Kräuterbüchern und an seine Darstellungsaufgaben gebunden.

Die mittel- und frühneuhochdeutschen Kräuterbücher bzw. Kräuterkapitel in Enzyklopädien oder Drogenkunden sind sowohl sprach- und textsortengeschichtlich (z.B. Habermann 2002a; 2002b; 2003; Gloning 2007; Seidensticker 1990; 1993; 1996; 1997; 2001; 2010), medizingeschichtlich (z.B. Schnell 1991; 1999; 2000; 2003; 2005; Keil 1980; 1982; 1983; 2002; Keil / Dilg 2003; Müller 2005), buch- und werkgeschichtlich (z.B. Hoppe 1969; Hayer 1998; Mayer 1995; 2000; Mayer / Goehl 2002; 2003; Riethe 2005) als auch editorisch (z.B. Schnell / Crossgrove 2003; Hayer / Schnell 2010; Hayer 1997; Anholter-Moyländer Kräuterbuch 2004; Fehringer 1994) insgesamt gut untersucht. Hinzu kommen Studien zur Bildnutzung in Kräuterbüchern (z.B. Baumann 1998; Gerhardt / Schnell 2002).

1 Ich widme diesen Beitrag Christel und Peter Seidensticker (Lahr) mit Dank für einen langjährigen Dialog und für vielfältige Unterstützung. So beruht etwa die digitale Transkription des $\mathrm{Ga}$ erde der suntheit, die wir für das Deutsche Textarchiv (DTA) aufbereitet haben, auf ihrer Arbeit. 
In jüngerer Zeit erleichtert vor allem die digitale Verfügbarkeit von Handschriften und Frühdrucken die Forschung in erheblichem Maße. Es steht aber außer Frage, dass auch im Bereich der mittel- und frühneuhochdeutschen Kräuter- und Medizintexte in sprachlicher und textueller Hinsicht noch viel zu tun ist.

In Bezug auf die mittelniederdeutschen Kräuterbuch-Texte bis zum Ende des 16. Jhs. ist die Forschungslage, jedenfalls soweit ich sehen kann, nicht ganz so gut. Immerhin gibt es aber auch hier Editionen (z.B. Seidensticker 1999) und einzelne Untersuchungen, wenn man aber die Überblicksartikel von Gundolf Keil zur ,Gart der gesuntheit‘- und zur ,Hortus sanitatis'-Tradition vor Augen hat, dann fehlen ähnlich detaillierte Übersichten für den mnd. Gaerde der suntheit. Der Gaerde wird im Keil-Beitrag von 1980 auf wenigen Zeilen mitbehandelt, ein für den Nachtragsband angekündigter Artikel zu Stephan bzw. Steffen Arndes ist dann aber im Bd. 11 des Verfasserlexikons mit „entfällt“ gekennzeichnet. Im Hinblick auf den allgemeineren medizinischen Sprachgebrauch kann man eine Reihe von Editionen und Untersuchungen zu mnd. Arzneibüchern mit heranziehen, die im Hinblick auf Aspekte wie den Wortgebrauch auch für die Kräutertexte aufschlussreich sind (z.B. Norrbom 1921; Windler 1932; Lindgren 1967; vgl. auch Temmen 1998; 2006).

Die mnd. Druck-Überlieferung von Kräuterbüchern bzw. Kräuterkapiteln setzt 1483 mit dem Promptuarium medicinae ein. Dieses Werk wurde in seiner ersten Ausgabe in Magdeburg bei Bartholomäus Ghotan gedruckt, eine zweite Ausgabe erschien 1488 in Lübeck bei Matthäus Brandis. ${ }^{2}$ Ein zweiter, etwas kleinerer Text wurde 1484 in Lübeck als Kapitel Van den kruderen in einer mnd. Ortolf-Fassung mit dem Titel Bock der arstedien van allen krankheyten gedruckt. ${ }^{3}$ Bei diesem Pflanzenkapitel handelt es sich aber nicht um einen Ortolftext, sondern um das Pflanzenkapitel aus Konrads von Megenberg Buch der Natur. Der Austausch hat aber offenbar schon in der vorgängigen hd. Drucküberlieferung stattgefunden.

Der umfangreichste mnd. Kräuterbuchtext ist zweifellos der Gaerde der suntheit (gedruckt 1492 in Lübeck bei Stefan Arndes) mit seinen fast 650 Folioseiten und über 200.000 laufenden Wortformen. Das Werk ist in seinem Kern eine umfangreiche Zusammenstellung von Pflanzenmonographien, in die auch einige Monographien zu nicht-pflanzlichen Drogen eingebaut sind. Der Kerntext wird umrahmt von kleineren Texten (Vorrede, Harnschautext, Register), der Globalaufbau wird in einer Passage der Vorrede folgendermaßen erläutert:

2 Zur Textgeschichte und zu den noch erhaltenen Exemplaren der beiden Drucke vgl. die Edition von Peter Seidensticker (1990); vgl. weiter Keil 1993.

3 Dieser Text ist im Exemplar der Staatsbibliothek Berlin digital verfügbar. 
Tा Dyt bok wert gedelet in viff deyl. II Dat erste is de vorrede. nu hyr beroret II Dat ander deyl is van den navolghenden kruden vnde ander creaturen krafft vnde doghet. in voruolginge des alphabetes. II Dat drudde wert syn eyn register van kruden to laxirende vnde to sterkende. Jtem van den wolrukenden. Jtem van deme gummy. Jtem van den vruchten. sade vnde wortelen. Jtem van eddelensteenen. Item van den deerten. vnde wat van en ghetelet wert. vnde alzo wat to arstedye denet ghemeynliken $|0005|$ |ा Dat verde deel van allen farwen des waters. vnde wat eine iewelke farwe bedudet. II Dat vefte deel vnde dat leste werd syn eyn regyster behende to vinden van allen ghebrecken vnde krankheiden des minschen wo de syn moghen. ${ }^{4}$

Nach den Angaben von Keil beruht der Text des mnd. Gaerde von 1492 im Wesentlichen auf dem Textbestand des fnhd. Gart der gesuntheit (Mainz: Schöffer 1485), es wurden aber auch Übersetzungen von Zusatzkapiteln eingefügt, die auf dem lateinischen Hortus sanitatis (Mainz: Meydenbach 1491) beruhen. Dieses lat. Werk war eine inhaltlich erweiterte Konkurrenzproduktion zum deutschen Gart der gesuntheit von 1485. Die insgesamt recht filigranen Fragen der Textentwicklung und der Zusammenhänge zwischen den unterschiedlichen Fassungen sind in den Beiträgen von Keil (1980; 1982; ${ }^{5}$ 1983) zusammenfassend und mit Rückgriff auf die älteren medizingeschichtlichen, buchgeschichtlichen, textgeschichtlichen, pharmakobotanischen usw. Forschungen dargestellt.

Insgesamt muss man - wie schon erwähnt - sagen, dass der Gaerde der suntheit von 1492 im Detail noch nicht sehr gut erforscht ist, auch im Vergleich mit anderen Texten aus der ,Gart' / ,Hortus'-Tradition.

Im vorliegenden Beitrag frage ich, wie man die textuelle Organisation und den Wortgebrauch der Kerntexte im Gaerde charakterisieren kann. Die Pflanzenmonographien im Gaerde sind, wie in vielen anderen Kräuterbüchern und

4 Die Seitenhinweise zwischen den || in den Zitaten beziehen sich auf die elektronische Fassung des Garde der sundheit im DTAQ-Bereich des Deutschen Textarchivs. Die Zahl bezieht sich auf die Nummer des jeweiligen Seitenscans nach dem Exemplar der SUB Göttingen. Zitate aus den Pflanzenmonographien zitiere ich im Folgenden mit der Kapitel-Nummer.

5 Der Beitrag von 1982 in der Daems-Festschrift entspricht in weiten Teilen und auch in der Struktur dem Artikel im Verfasserlexikon von 1980. Im Festschriftenbeitrag findet man allerdings auf S. 609 eine kurze Ausführung zu Steffen Arndes, dem Lübecker Drucker des Gaerde; wie schon erwähnt, wird im Verfasserlexikon-Artikel von 1980 in Spalte II, 1084 auf einen eigenen Artikel zu Steffen Arndes im Nachtragsband verwiesen. Im Nachtrags-Band 11 (2004) heißt es dann aber nur kurz: „Arndes, Stephan entfällt (vgl. $\rightarrow$...)“, der Verweis nennt dann die beiden Keil-Artikel zum Gart und zum Hortus sanitatis in den Bänden II bzw. IV. - Zur Art der Bearbeitung im Gaerde schreibt Keil nur: „Steffen Arndes hat den ,Gart“ ins Nd. umgeschrieben und die aus dem ,Hortus‘ entlehnten Auszüge in Lübecker Mundart übersetzt“ (1982, 609). Es bleibt zu fragen, wie sich das Umschreiben zum Übersetzen verhält, wie das Nd. zur Lübecker Mundart (ist im Text ein Unterschied in den entsprechenden Passagen erkennbar?), es fragt sich auch, ob Arndes den Text selbst hergestellt hat und woher man das weiß. 
Simplizienverzeichnissen, hochgradig schematisch organisiert. Deshalb kann die Analyse eines typischen Beispiels bereits Aufschlüsse über grundlegende sprachliche und textuelle Eigenschaften geben. Ich wähle für eine exemplarische Betrachtung die Pflanzenmonographie zur Absinth-Pflanze, die unten abgedruckt wird. In der folgenden Darstellung kombiniere ich die Befunde der exemplarischen Untersuchung zur Absinthium-Monographie mit Befunden aus dem weiteren Text. Es folgt also zunächst der Beispieltext (Abschnitt 2), sodann die Ausführungen zur Textorganisation (Abschnitt 3) und zum Wortgebrauch (Abschnitt 4). Abschließend folgen Perspektiven für die weitere Arbeit.

\section{Ein Textbeispiel: „Absinthium wormede Cap. iii.“}

\subsection{Abbildung des Absinth-Kapitels}

Die folgende Doppelseite aus dem Gaerde soll einen Eindruck vermitteln von der typographischen Gestaltung des Buches, diese Seiten zeigen auch die handschriftlichen Marginalien, die im Göttinger Exemplar auf einigen Seiten angebracht wurden.

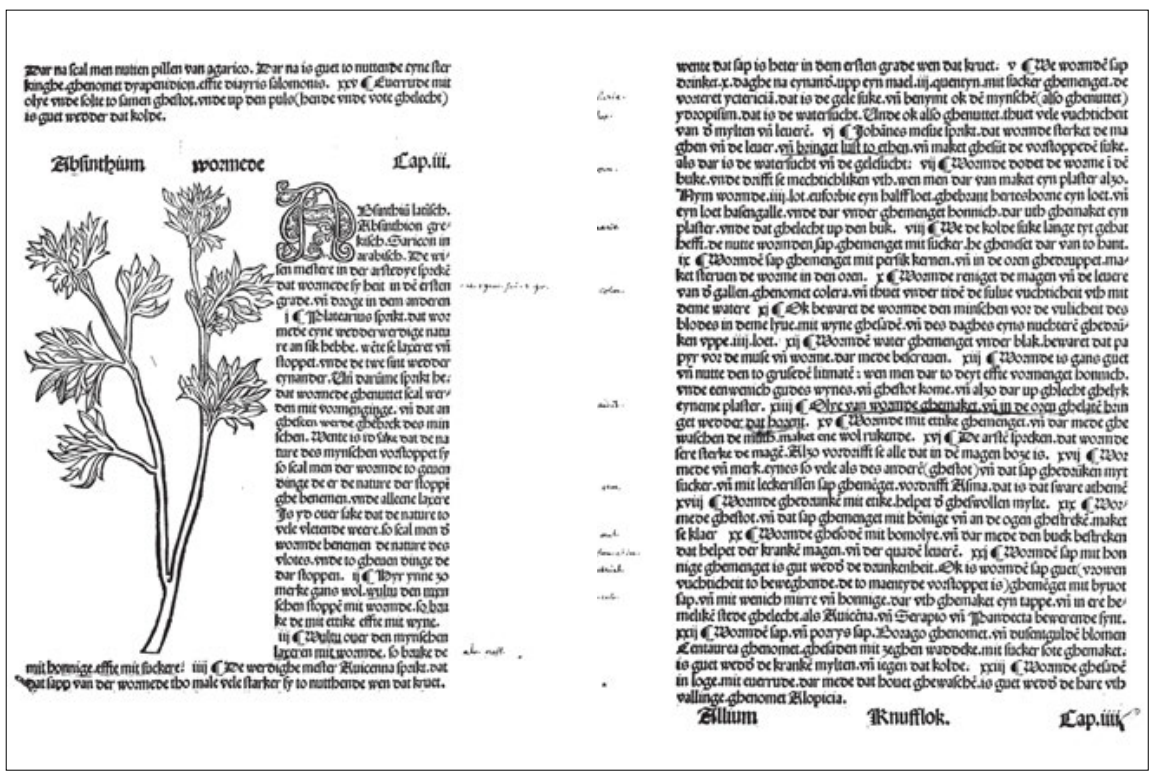

Abb. 1: Gaerde der suntheit 1492, Kap. 3 (SUB Göttingen, 4 MAT MED 38 / 19 INC) 
Die Bilddigitalisate des Göttinger Exemplars sind im Deutschen Textarchiv zusammen mit der Arbeitstranskription verfügbar, ${ }^{6}$ hier zum Beispiel die seitensynoptische Ansicht. Die Arbeitstranskription kann in dieser Umgebung auch korrigiert und in unterschiedlichen Fassungen heruntergeladen werden.

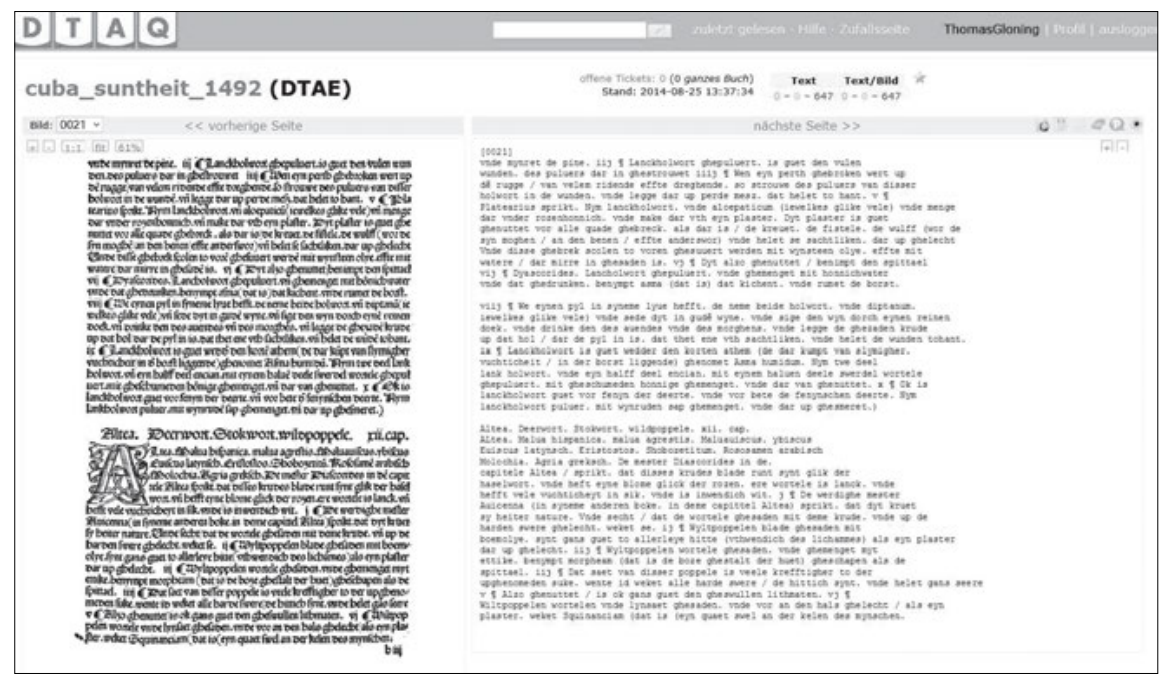

Abb. 2: Screenshot Deutsches Textarchiv, Bereich DTAQ.

\subsection{Transkription}

Absinthium wormede Cap. iii.

[Abbildung]

ABsinthium latinsch. Absinthion grekisch. Saricon in arabisch. De wisen mestere in der arstedye spreken dat wormede sy heit in deme ersten grade. vnde droge in dem anderen $j$ II Platearius sprikt. dat wormede eyne wedderwerdige nature an sik hebbe. wente se laxeret vnde stoppet. vnde de twe sint wedder eynander. Vnde darumme sprikt he. dat wormede ghenuttet scal werden mit vormenginge. vnde dat angheseen werde ghebrek des minschen. Wente is id sake dat de nature des mynschen vorstoppet sy so scal men der wormde to geuen dinge de er de nature der stoppinghe benemen. vnde alleene laxere Is yd ouer sake dat de nature to vele vletende weere. so scal men der wormde benemen de nature des vlotes. vnde to gheuen dinge de dar stoppen. ij. II Hyr ynne zo merke gans wol. wultu den mynschen stoppen mit wormde. so bruke de mit ettike effte mit wyne.

6 Online verfügbar unter http://www.deutschestextarchiv.de/dtaq/book/show/cuba_suntheit_ 1492 (letzter Aufruf 16.4.2015). Für den DTAQ-Bereich ist eine unkomplizierte Online-Registrierung erforderlich. 
iij Tा Wultu ouer den mynschen laxeren mit wormede. so bruke de mit honnige. effte mit suckere. iiij II De werdighe mester Auicenna sprikt. dat dat sapp van der wormede tho male vele starker sy to nutthende wen dat kruet. /0010/ wente dat sap is heter in dem ersten grade wen dat kruet. $v$ 9ा We wormden sap drinket. $x$. daghe na eynander. upp eyn mael. iij. quentyn. mit sucker ghemenget. de vorteret yctericiam. dat is de gele suke. vnde benymt ok deme mynschen (also ghenuttet. ydropisim. dat is de watersucht. Vnde ok also ghenuttet. thuet vele vuchticheit van der mylten vnde leueren. vj II Johannes mesue sprikt. dat wormde sterket de maghen vnde de leuer. vnde bringet lust to ethen. vnde maket ghesunt de vorstoppeden suke. als dar is de watersucht vnde de gelesucht: vij 9 Wormde dodet de worme in deme buke. vnde drifft se mechtichliken vth. wen men dar van maket eyn plaster alzo. Nym wormde. iiij. lot. euforbie eyn halff loet. ghebrant herteshorne eyn loet. vnde eyn loet hasengalle. vnde dar vnder ghemenget honnich. dar uth ghemaket eyn plaster. vnde dat ghelecht up den buk. viij. II We de kolde suke lange tyt gehat hefft. de nutte wormden sap. ghemenget mit sucker. he gheneset dar van to hant. ix II Wormden sap ghemenget mit persik kernen. vnde in de oren ghedruppet. maket steruen de worme in den oren. $x$ II Wormde reniget de magen vnde de leuere van der gallen. ghenomet colera: vnde thuet vnder tiden de sulue vuchticheit vth mit deme water xj II Ok bewaret de wormde den minschen vor de vulicheit des blodes in deme lyue. mit wyne ghesaden. vnde des daghes eyns nuchteren ghedrunken vppe. iiij. loet. xij 9 I Wormden water ghemenget vnder blak. bewaret dat papyr vor de muse vnde worme. dar mede bescreuen. xiij II Wormde is gans guet vnde nutte den to gruseden litmaten: wen men dar to deyt effte vormenget honnich. vnde eenwenich gudes wynes. vnde ghestot kome. vnde alzo dar up ghelecht ghelyk eyneme plaster. xiiij TI Olye van wormde ghemaket. vnde in de oren ghelaten bringet wedder dat horent. xv II Wormde mit ettike ghemenget. vnde dar mede ghewaschen de munth. maket ene wol rukende. xvj 9 I De arsten spreken. dat wormde sere sterke deme magen. Alzo vordriff se alle dat in den magen boze is. xvij 9 II Wormede vnde merk. eynes so vele als des anderen (ghestot) vnde dat sap ghedrunken myt sucker. vnde mit leckerissen sap ghemenget. vordrifft Asma. dat is dat sware athemen xviij 9 I Wormde ghedrunken mit etike. helpet der gheswollen mylte. xix II Wormede ghestot. vnde dat sap ghemenget mit honnige vnde an de ogen ghestreken. maket se klar xx II Wormde ghesoden mit bomolye. vnde dar mede den buek bestreken dat helpet der kranken magen. vnde der quaden leueren. xxj Tा Wormden sap mit honnige ghemenget is gut wedder de drunkenheit. Ok is wormden sap guet (vrowen vuchticheit to beweghende. de to maentyde vorstoppet is) ghemenget mit byuot sap. vnde mit wenich mirre vnde honnige. dar vth ghemaket eyn tappe. vnde in ere hemeliken stede ghelecht. als Auicenna. vnde Serapio vnde Pandecta bewerende synt. xxij Tा Wormden sap. vnde porrys sap. Borago ghenomet. vnde dusentgulden blomen Centaurea ghenomet. ghesaden mit zeghen waddeke. mit sucker sote ghemaket. is guet wedder de kranken mylten. vnde iegen dat kolde. xxiij II Wormde ghesaden in loge. mit euerrude. dar mede dat houet ghewaschen. is guet wedder de hare vthvallinge. ghenomet Alopicia.

\section{Textorganisation}

Wie oben schon angedeutet, besteht der Gaerde der suntheit aus fünf größeren Teilen: Vorrede, Kräuterbuch, Steinbuch, Harnschautext, Register. Mit all diesen Teilen sind auch spezifische Darstellungsformen verbunden: Im Kräuterbuch 
und im Steinbuch ist die Drogen-Monographie die zentrale Darstellungsform, der Harnschautext hat Abhandlungscharakter, die Register sind je nach Funktion unterschiedlich aufgebaut. Da das Kräuterbuch der zentrale Text ist, stelle ich in diesem Abschnitt die textuelle Form der Pflanzenmonographie in den Mittelpunkt.

\subsection{Pflanzenmonographien: das textuelle Makro-Schema}

Die Pflanzenmonographie zur Absinth-Pflanze zeigt ein Schema, das vier Textteile umfasst, die sich im Hinblick auf Funktionen und Teilthemen näher bestimmen lassen.

(i) Die Überschrift gibt an, um welche Pflanze es in der Monographie geht. Die Überschrift umfasst ihrerseits drei Informationspositionen: die lat. Pflanzenbezeichnung, die auch die Grundlage ist für die halbalphabetische Anordnung der Monographien, sodann die volkssprachige Bezeichnung (ggf. auch mehrere Bezeichnungen) und die Kapitelnummer.

Absinthium wormede Cap. iii.

Die Kapitelnummer erfüllt eine wichtige Funktion für die Erschließung des Textes mit Hilfe von Registern. Im Krankheiten- bzw. Beschwerdenregister werden die unterschiedlichen Heilanzeigen mit der Kapitelnummer und der kapitelinternen Abschnitt-Nummer der betreffenden Heilanzeige angegeben.

(ii) Der zweite Textteil umfasst nur wenige Zeilen, hier werden die lat., griech. und arab. Bezeichnungen der Pflanze genannt und aufeinander bezogen. Man erkennt hier deutlich ein funktional geprägtes syntaktisches Muster, bei dem die Pflanzenbezeichnung mit einer nachgestellten Sprachbezeichnung kombiniert wird.

ABsinthium latinsch. Absinthion grekisch. Saricon in arabisch.

Auch diese Praxis hat eine lange Tradition in der pharmakologischen Literatur. Sie diente unter anderem dazu, entsprechende Parallelstellen in den autoritativen Texten der griech. (z.B. Dioskurides), lat. (z.B. Macer) oder arab. Tradition (z.B. Avicenna), auch wenn letztere vorwiegend in lat. Übersetzungen genutzt wurden, auffinden zu können.

(iii) Im dritten Textteil unseres Beispiels wird die humorale Natur der Pflanze im System der Vier-Säfte-Lehre charakterisiert, indem der Pflanze in den beiden Dimensionen heiß / kalt und feucht / trocken jeweils eine Primärqualität (z.B. heit 'humoral heiß', droge 'humoral trocken') zugeschrieben wird. Die Primär- 
qualität wird darüber hinaus im Hinblick auf ihre Intensität auf einer Stufenskala näher spezifiziert.

De wisen mestere in der arstedye spreken dat wormede sy heit in deme ersten grade. vnde droge in dem anderen

Zugrunde liegt hier die Gradus-Lehre, wie sie seit dem Mittelalter etwa im Liber de gradibus, einer Übersetzung des Constantinus Africanus, bis in die Frühe Neuzeit hinein gewirkt hat. Da in unserem Beispiel nur der erste und der zweite Intensitätsgrad erwähnt werden, könnte man an dieser Stelle noch nicht sagen, ob eine Dreier- oder eine Vierer-Skala angenommen wird. Es gibt aber eine ganze Reihe von Stellen, z.B. die folgende aus dem Kap. 8, in denen der vierte Grad zugesprochen wird, im Text wird also eine Viererskala verwendet:

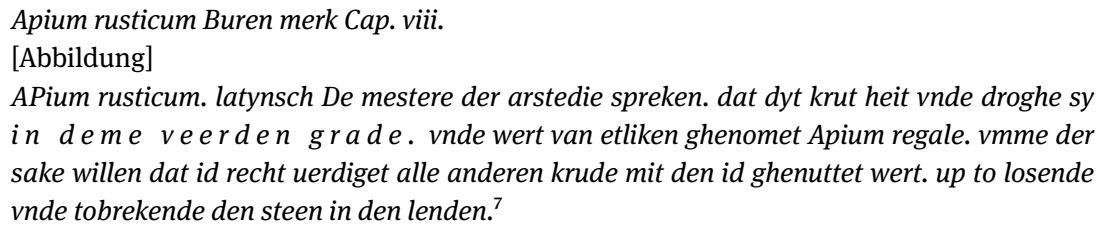
in de me veerden grade. vnde wert van etliken ghenomet Apium regale. vmme der sake willen dat id recht uerdiget alle anderen krude mit den id ghenuttet wert. up to losende vnde tobrekende den steen in den lenden. ${ }^{7}$

In der Absinth-Monographie gibt es im vierten Text-Teil noch weitere humorale Charakterisierungen, die sich dann aber auf bestimmte Pflanzenteile im Kontext medizinischer Anwendungsfragen beziehen (z.B. im Teilabschnitt iiij). Auch in anderen Monographien werden zuweilen einzelne Pflanzenteile charakterisiert, dann allerdings im Kontext bestimmter medizinischer Anwendungen. Im nächsten Beispiel sieht man, dass der Textteil zur humoralen Charakterisierung der Pflanze Teil der Nummerierung ist:

\footnotetext{
Alkekengi Ioden kerse. effte Boberellen xxiiii. cap.

ALkekengi latynsch vnde grekisch. $j$ II De mester Serapio sprikt. dat disses krudes doghet sy in der vrucht vnde nicht an deme krude De vrucht is gheschapen als eyne kerssebere. vnde is roet. vnde hefft eyne huet dar ouer glik als de spinnewobbe. vnde de vrucht is droghe vnde kolt an dẽ anderen grade. ij II Dyascorides sprikt. [...]
}

Schließlich begegnen auch Fälle, in denen die Autoritäten konkurrierende Angaben zur humoralen Charakteristik machen. Im folgenden Beispiel werden etwa die Angaben aus dem Circa instans und dem sog. Pandectarius als nicht übereinstimmend kontrastiert:

7 Sperrung hier und in allen folgenden Belegen von Thomas Gloning. 
Accacia Sleesap. effte vnripe plumen sap xxvi. cap

[Abbildung]

Accacia latinsch. vnde grekisch. Altarti arabisch. In dem boke circa instans ghenomet in dem capittel Accacia / steit ghescreuen dat de Sleesap sy kold vnde droge in dẽ drudden grade. P a n d e c t a s p ri $k t$. dat de sleesap sy kold in de. anderen grade. vnde droge in dem drudden grade.

Aber auch die Übereinstimmung von Autoritäten kann explizit erwähnt werden:

Aloes lignum Aloes holt. xxxvi. cap.

[Abbildung]

ALoes lignum. effte xiloaloes latinisch. Aloa. effte Agalaym grekisch. Hoad arabisch. De werdighe mester Auicenna in sineme anderen boke in dẽ capittel xiloaloes. ok de mester Rasis. des geliken de mester ysaac vnde Serapio vnde Platearius spreken alle samentliken. dat aloes holt sy heit vnde droge an deme anderen grade.

Weiterhin gibt es Beispiele, in denen botanische Eigenschaften einer Pflanze beschrieben werden, entweder zusätzlich zur oder anstelle der humoralen Charakterisierung:

Aristologia longa Lanck holwoert xi. cap.

[Abbildung]

Aristologia longa latynsch. De werdigen mestere Auicenna. Galienus Diascorides Platearius vnde Plinius spreken dat de lange holwort sy warm in dẽ drudden grade. vnde droge in dê anderen grade Disse holwort hefft eynen langen stengel vnde blade dar an de gliken den bladen an der haselwort. men de blade der langen holwort synt weker an dẽ grepe De worttele is lanck glick der petersilligen wortele. [...]

Im folgenden Beispiel wird die Pflanze nur botanisch charakterisiert, humorale Eigenschaften werden erst im Teil zur medizinischen Nutzung thematisiert.

Altea. Deerwort. Stokwort. wildpoppele. xii. cap.

ALtea. Malua hispanica. malua agrestis. Maluauiscus. ybiscus Euiscus latynsch. Eristostos. Shobozetitum. Rososamen arabisch Molochia. Agria greksch. De mester Diascorides in de capitele Altea / sprikt. dat disses krudes blade runt synt glik der haselwort. vnde heft eyne blome glick der rozen. ere wortele is lanck. vnde hefft vele vuchticheyt in sik. vnde is inwendich wit. II De werdighe mester Auicenna (in syneme anderen boke. in deme capittel Altea) sprikt. dat dyt kruet sy heiter nature. Vnde secht / dat de wortele ghesaden mit deme krude. vnde up de harden swere ghelecht. weket se. [...]

(iv) Der vierte und längste Makro-Textteil der Monographie ist eine strukturierte Besprechung von Aspekten der medizinischen Nutzung der betreffenden Pflanze. Der Beginn wird im Absinth-Beispieltext durch eine kapitelinterne Zählung mit römischen Kleinbuchstaben eingeleitet. Im Wesentlichen besteht dieser MakroTextteil aus einer Aufzählung unterschiedlicher Heilanzeigen, die typischerweise 
aus drei Teilbausteinen bestehen, mit denen jeweils die Zubereitung, die Art der Anwendung und die medizinische Wirkung beschrieben werden (siehe hierzu Abschnitt 3.2.1).

Man kann verallgemeinern, dass die Pflanzenmonographien im Gaerde folgende funktional-thematischen Schwerpunkte aufweisen:

- Adressierung der Pflanzen mit lateinisch-deutscher Pflanzenbezeichnung und Kapitelnummer;

- Bezeichnungen der Pflanze in weiteren Sprachen;

- humorale und ggf. botanische Charakteristik der Pflanze;

- medizinisches Profil und Heilanzeigen der Pflanze.

Diese thematischen Schwerpunkte sind aber nicht immer säuberlich auf vier abgrenzbare Makro-Textteile beziehbar. In den Abschnitten zu Heilanzeigen können auch Fragen der humoralen Charakteristik oder botanische Aspekte der Pflanze diskutiert werden, sofern sie für die medizinische Nutzung der Pflanze erheblich sind.

Auch die Nummerierung ist kein eindeutiges Signal, dass der Bereich der Heilanzeigen beginnt, auch wenn das in vielen Monographien zutrifft. Im Kapitel 46 (Aspergula) zum Beispiel, das nicht auf den hd. Gart, sondern auf den lat. Hortus von 1491 zurückgeht, sind auch die Ausführungen zum Habitus und zur humoralen Charakteristik der Pflanze Teil der Nummerierung. Auch im darauf folgenden Kapitel 47 (Auellana Jndica) sind Ausführungen zur Charakterisierung der Pflanze schon nummeriert, die im Hortus von 1491 unter „Operationes“ zusammengestellten Heilanzeigen beginnen im Gaerde bereits mit der Ziffer iiij. Im hd. Gart von 1485 gibt es kein Nummerierungssystem für die Heilanzeigen, sie sind jeweils nur durch Alinea-Zeichen abgesetzt. Die Nummerierung und die thematische Makrostrukturierung sind also nicht immer parallel geführt, obwohl das im Prinzip möglich gewesen wäre, die Heilanzeigen im Hortus sind sogar zusätzlich durch eine eigene Überschrift („Operationes“) abgesetzt und durch Großbuchstaben als textuelle Einheiten markiert.

Wir verlassen nun die globalen Prinzipien der textuell-thematischen Schematisierung von Drogenmonographien im Gaerde und wenden uns einzelnen funktionalen Textbausteinen $\mathrm{zu}$. 


\subsection{Funktionale Textbausteine}

Funktionale Textbausteine sind typische und häufig wiederkehrende Textteile, mit denen bestimmte Teil-Funktionen realisiert werden können. Sie hängen besonders eng mit den spezifischen Darstellungsaufgaben im Rahmen eines Texttyps, hier der Pflanzenmonographie, zusammen und weisen in der Regel ein mehr oder weniger breites Repertoire von syntaktisch-lexikalischen Realisierungsformen auf (vgl. Gloning 2010; Fritz 2013, Kap. 2.1). Oben wurde bereits eine Reihe von funktionalen Textbausteinen kommentiert, z.B. solche mit der Teilfunktion ,die humorale Natur einer Pflanze im System der Vier-Säfte-Lehre charakterisieren` oder ,die unterschiedlichen Bezeichnungen für eine Pflanze angeben‘.

\subsubsection{Der Textbaustein ,Heilanzeige‘}

Heilanzeigen sind der zentrale Textbaustein in Kräuterbüchern, mit ihnen wird das medizinische Heilpotenzial einer Pflanze in strukturierter Form beschrieben. Die einzelnen Textportionen, in denen spezifische Heilanzeigen beschrieben werden, sind über die kapitelinterne Nummerierung gezielt aus dem Krankheitsregister heraus anzusteuern.

Die Formulierung einer einzelnen Heilanzeige weist in der Regel drei elementare Bestandteile auf: (a) Angaben zur Art der Herstellung des Heilmittels, (b) Angaben zur Art der Anwendung des Heilmittels und (c) Angaben zur medizinischen Wirkung nach der Anwendung. Ein einfaches Beispiel, bei dem die Bestandteile gut erkennbar sind, ist Teilabschnitt ix aus der Absinth-Monographie:

ix $\mathbf{~ I I ~ W o r m d e n ~ s a p ~ g h e m e n g e t ~ m i t ~ p e r s i k ~ k e r n e n . ~ v n d e ~ i n ~ d e ~ o r e n ~ g h e d r u p p e t . ~ m a k e t ~ s t e r u e n ~}$ de worme in den oren.

In der folgenden Version sind die drei Textbausteine durch eckige Klammern abgegrenzt und mit (a)-(c) markiert:

ix $\boldsymbol{~}$ (a) [Wormden sap ghemenget mit persik kernen.] vnde (b) [in de oren ghedruppet.] (c) [maket steruen de worme in den oren.]

Der Textbaustein Heilanzeige weist insgesamt eine gewisse Variation auf, sowohl was die Abfolge der drei Bestandteile als auch was die syntaktischen Realisierungsmuster betrifft. Das folgende Beispiel aus dem ersten Kapitel (Artemisia) beginnt nach einer Quellenkennzeichnung mit der medizinischen Wirkung, dann folgt die Zubereitung, zum Schluss folgt die Art der Anwendung: 
xiiij Фा Diascorides sprikt. Byuoet is gans guet vor den steen. wen men den puluert. vnde dat puluer menget mit merk water. vnde dat alzo drinket.

Die Nutzung von einfachen oder komplexeren Quellenangaben ist eine der Anreicherungsmöglicheiten von Heilanzeigen (Beispiele aus Kap. 2):

ix II Pla te a rius sprikt. Euerrude ghesaden mit bomolye. vnde dat houet dar mede bestreken. benympt de kolde des houedes. vnde wert warm dar van.

iiij Фा Ok sprikt Auicenna in synem. ij. boke in deme capitel Abrot a n u m. dat Euerrude ghebrant vnde ghepuluert. vnde dat ghemenget mit reddikolye. vnde dat ghesmeret up de kale stede. maket hare wassen.

Zum Variationsspektrum gehört auch die Möglichkeit, weitere Textbausteine einzulagern. Eingelagert in die Heilanzeige des Abschnitts vij im Absinth-Kapitel ist zum Beispiel ein Rezept für die Herstellung eines Pflasters. Aufhänger dafür ist der Gebrauch des Ausdrucks plaster im Rahmen der Heilanzeige. Die Herstellungsanleitung wird mit einem Vorverweis angekündigt (wen men dar van maket eyn plaster alzo). Die Herstellungsanleitung endet dann mit dar uth ghemaket eyn plaster, um dann die eigentliche Heilanzeige fortzusetzen, die mit der Angabe zur Anwendung schließt (vnde dat ghelecht up den buk).

Die drei Teilbausteine einer Heilanzeige (Zubereitung, Art der Anwendung, medizinische Wirkung) weisen ein insgesamt stark schematisiertes syntaktisches Repertoire auf. Zubereitung und Art der Anwendung werden z.B. häufig mit Hilfe partizipialer Konstruktionen ausgedrückt: Wormden sap ghemenget mit persik kernen. vnd in de oren ghedruppet [...]. Für die Charakterisierung einer medizinischen Wirkung bzw. eines medizinischen Anwendungsbereichs werden vor allem zwei syntaktische Muster genutzt, die intern eine beträchtliche Realisierungsbreite aufweisen:

Nominalgruppen für Personen mit einem bestimmten Beschwerdebild (We de kolde suke lange tyt gehat hefft [...]; We beuallen were mit bozer kolden vuchticheit. ynwendich effte vthwendich des lychams [...]);

Prädikationen, die aus einem verbalen Bestandteil und einer elementaren oder komplexen Bezeichnung für ein Beschwerdebild, ein Organ, für einen positiven medizinischen Effekt usw. bestehen (vordrifft Asma; benimpt peripleumoniam; is guet wedder de kranken mylten; bringet wedder dat horent; heylet boze swere).

Diese Konstruktionsmuster, deren Realisierungsspielräume und Häufigkeitsverteilung an anderer Stelle weiter verfolgt werden müssen, können auch kombiniert werden (Beispiel aus Kap. 4):

[...] vordriuet dat lenden wee. vnd helpet ok deme de dar swarlick atempt. 
Insgesamt kann man festhalten: Die Formulierung der Heilanzeigen weist einerseits einen beträchtlichen Grad der Schematisierung auf, zum anderen aber auch ein gewisses Ausmaß an Variation in der syntaktisch-lexikalischen Umsetzung und in der Abfolge der Bestandteile. Art und Grad der Schematisierung mitsamt den Realisierungsspielräumen bewegen sich im Rahmen dessen, was in hochund spätmittelalterlichen Pflanzenmonographien üblich war.

\subsubsection{Formen der Quellenkennzeichnung}

Formen der Quellenkennzeichnung gehören $\mathrm{zu}$ den wesentlichen sprachlichen Verfahren in Texten der älteren Medizin (vgl. Gloning 2011a). Sie hängen eng zusammen mit einem Wissenschaftsverständnis, das Autoritäten und ihren Texten eine wesentliche Rolle bei der Sicherung und Darstellung wissenschaftlicher Erkenntnis zuschrieb. Wer Wissenschaft treiben wollte, musste die alten Autoritäten und ihre Positionen kennen und anführen können, sei es aufgrund der originalen Texte, soweit sie in der Überlieferung verfügbar waren, sei es aufgrund von sekundären Quellen.

Im Gaerde werden Angaben zur humoralen oder (seltener) botanischen Charakteristik von Pflanzen und vor allem auch Angaben zu Heilanzeigen immer wieder mit Formen der Quellenkennzeichnung kombiniert. Die elementare und am häufigsten genutzte Form dafür ist ein Matrixsatz, der aus der Nennung einer Autorität und einem Verbum dicendi (z.B. sprikt) besteht, z.B. Dyascorides sprikt (Kap. 5), De wyse mester Galienus sprikt (Kap. 5), Auicenna sprikt (Kap. 6). Neben namentlich genannten Autoren begegnen auch unspezifische Hinweise auf medizinische Fachleute, z.B. De mestere der arstedie spreken. dat dyt krut heit vnde droghe sy in deme veerden grade (Kap. 8).

Quellen- und Autoritätenkennzeichungen können auch erweitert werden mit Hinweisen auf die Fundstelle in einem Text. Im folgenden Beispiel aus dem Gaerde wird das Muster <autor> + sprikt angereichert mit Angaben zum Werktitel und zur Fundstelle im Text, die Verbform des Inhaltssatzes steht im Indikativ. Eine interessante Frage ist dabei auch, wie weit sich der Geltungsbereich der Quellenangabe erstreckt. Erstreckt sie sich nur auf den ersten Satz oder kann man sie auch auf die Folgeangaben beziehen? Syntaktisch lässt sich diese Frage nicht entscheiden, man müsste hier die erwähnten Quellentexte daraufhin überprüfen, ob die wiedergegebenen Aussagen gedeckt sind.

$v$ ๆा De mester ysaac (in syneme boke ghenomet De dietis particularibus in dem capitel Apium) sprikt. dat merk ghemenget mit wyne. sote ghemaket mit honnige ghenomet mellecraet. maket wol waterlaten. Vnde alzo ghenuttet / bringet ok de krankheit der vrouwen / menstruum ghenomet. Merk alzo ghenuttet. maket wol douwen. (Kap. 6, Abschnitt v) 
Neben diesen erweiterten Formen gibt es umgekehrt aber auch Fälle, in denen die Quellenkennzeichnung auf die Nennung der Autorität beschränkt ist:

vij \ा Dyascorides. Lancholwort ghepuluert. vnde ghemenget mit honnichwater vnde dat ghedrunken. benympt asma (dat is) dat kichent. vnde rumet de borst.

Es gibt Hinweise darauf, dass die Quellenkennzeichnungen bereits in einer textuellen Vorlage gestanden haben und dass der Autoritätenbezug damit sekundär ist. Hierfür ein Beispiel aus dem Knoblauch-Kapitel des Gaerde, das Parallelen zum Deutschen Macer aufweist:

\begin{abstract}
xx Фा Pitagoras sprikt. Knuflok ghestot. dar vnder ghemenget coriander. vnde also ghenuttet mit wine. vordriuet dat lenden wee. vnde helpet ok deme de dar swarlick atempt. also ghenuttet. De sulue drank weket den buck.
\end{abstract}

Pitagoras, ein meister, sagt: knoblouch gestossen mit coriandre unde mit wine unt das gedrungen unde genutzet, vertribet ein suche, die heiset neufresis, das ist zu dỉte lendensuche, und hilfet auch dem, der swere edemt. Der selbe tranc weichet den buch. (Schnell / Crossgrove 2003, 331). ${ }^{8}$

Insgesamt lässt sich auch hier festhalten, dass die im Gaerde genutzten Verfahren und Muster der Quellenkennzeichnung sich im Rahmen des zeitgenössischen Repertoires bewegen, das in der oben genannten Untersuchung (Gloning 2011a) erhoben ist. Und: Wenn man auf diese Formen der Quellen- und Autoritätenkennzeichnung verweist, muss man andererseits aber auch sagen, dass weite Teile des Gaerde ungekennzeichnet sind, also keinen Hinweis auf Quellen enthalten.

Darüber hinaus sind im Gaerde eine Vielzahl weiterer funktionaler Textbausteine zu belegen, z.B. Querverweise, Formen der Begründung oder terminologische Einführungen, sie können hier aber nicht näher kommentiert werden.

\title{
4 Aspekte des Wortgebrauchsprofils
}

Im Hinblick auf einen Fachtext dieser Art stellen sich u.a. folgende Fragen: Wie hängt der Wortgebrauch mit den thematischen und textfunktionalen Aufgaben der Darstellung zusammen? Inwiefern ist der mnd. Wortgebrauch des Textes

8 Im hd. Gart lautet diese Stelle: Jtem pitagoras ein meister spricht das knoblauch gestoßen dar vnder gemischet coriander vnd also genutzet mit win verdribet das lenden wee. vnd hilffet auch dem der da swere edemet also genutzet. Der selbige dranck weychet den buch (Kap. 4). Diese Fassung ist im Hinblick auf die Quellenkennzeichnung also noch etwas näher am Macer. 
fachlich geprägt, z.B. durch die Vier-Säfte-Lehre? Welche Rolle spielt die Wortbildung für die Bewältigung von Darstellungsaufgaben? Welche Rolle spielen die textuellen Vorlagen und die dt.-lat. Konstellation für den Wortgebrauch?

\subsection{Thematischer und fachlicher Wortgebrauch}

Die übergeordnete Funktion von Pflanzenmonographien ist es, das Heilpotenzial von Pflanzen zu charakterisieren. Diese Funktion bedingt die Notwendigkeit, Wörter in bestimmten Verwendungsweisen einzusetzen, die sich spezifisch medizinisch-pharmakologischen Wortschatzsektoren zuordnen lassen. Ich möchte diesen Bezug exemplarisch an drei Wortschatzsektoren verdeutlichen.

Ein erster Wortschatzsektor umfasst Verwendungsweisen, die sich dem Denksystem der Vier-Säfte-Lehre zuordnen lassen. Hierzu gehören zum einen Bezeichnungen wie heit 'humoral heiß, humoral warm', droghe 'humoral trocken', kolt 'humoral kalt' und vuchte 'humoral feucht'. Aber auch Ausdrücke wie nature 'humorale Komplexion', grade 'Ausprägungsgrad einer Elementarqualität auf einer Dreier- bzw. Vierer-Skala' oder vuchticheit 'Körpersaft' sind nur vor dem Hintergrund dieser medizinischen Lehre verstehbar. Das gilt auch für Verben, die eine spezifische Sekundärqualität im System der Säftelehre bezeichnen, z.B. laxeren, uth drifen oder stoppen, und für Bezeichnungen für eine physiologische Erscheinung im Rahmen der Säftelehre wie z.B. vorstoppinge. All diese Verwendungsweisen setzen das Denksystem der Säftelehre voraus und sind nur holistisch in ihrem wechselseitigen Zusammenhang erklärbar (vgl. Gloning 2011b).

Ein zweiter breit ausgebauter Wortschatzsektor sind Bezeichnungen für Krankheiten, Beschwerdebilder, (gestörte) Körperfunktionen und das Heilgeschehen im weitesten Sinne. ${ }^{9} \mathrm{Zu}$ den Krankheits- bzw. Beschwerdebezeichnungen gehören zum Beispiel Ausdrücke wie watersucht, fistel, fikblader, krevet 'fressendes Geschwür' oder waerte 'Warze'. Diese Funktion ist nicht immer lexikalisch, sondern häufig auch mit Mehrwort-Verbindungen realisiert, z.B. gheswollen mylte, vallende suke ‘Epilepsie' oder wedage des hovedes 'Kopfschmerzen' . Auch generalisierende Umschreibungen für größere Beschwerdebilder werden genutzt (z.B. [...] alle weedage der blazen. vnde der lenden; Kap. 351). Auch die schon erwähnten Personencharakterisierungen für Arten von Kranken (z.B. den de nicht waterlaten konen; Kap. 6) konkurrieren mit lexikalisierten Einheiten für Krankheiten und Beschwerdebilder.

9 Zur Frühgeschichte des medizinisch-heilkundlichen Wortgebrauchs vgl. vor allem Riecke (2004). 
Es ist eine eigene Frage, ob und wie aufgrund der sprachlichen Darstellung historische Krankheitskonzepte und Beschwerdebilder identifiziert und abgegrenzt werden können. Es ist klar, dass dabei die zeitgenössischen Wissensbestände und Bezugssysteme zugrunde gelegt werden müssen, was aber genau zum Beispiel mit krevet gemeint ist, ist schwer $\mathrm{zu}$ rekonstruieren. Es ist dabei hilfreich, textuelle Hinweise wie z.B. vnde is argher wen de kreuet (ok nomet me dat den wulff) (Kap. 374) auszuwerten, aber insgesamt bleibt man dabei doch in einem System wechselseitiger Abhängigkeiten und Bestimmungen, die nicht einfach zu rekonstruieren und die auch nicht leicht auf moderne Anschauungen zu beziehen sind. Die textuelle Umgebung im Kap. 374 zum Beispiel setzt drei Krankheitsbilder zueinander in Beziehung, zu deren Bezeichnungen es jeweils auch moderne Gegenstücke gibt (Herpes, Krebs, Wolf). Aber schon die Erklärung der Ätiologie (kumpt van der vorbranden colera) zeigt, dass die Konzeptualisierung in einem ganz anderen Rahmen stattfindet:

viij पा Dyt sap alzo temperet benimpt ok dat ghebrek Herpetes (effte herpestiomenus) ghenomet. dat is eyn swer de dat vlesch an de lichamme vorteret. vnde kumpt van der vorbranden colera. vnde is argher wen de kreuet (ok nomet me dat den wulff) dat sap dar upp ghesmeret vnde ghelecht.

Durch den gesamten Text ziehen sich Formen der Parallelisierung von mnd. und lat. Bezeichnungen, die entweder mit dat is oder mit einer ghenomet-Konstruktion verbunden werden, z.B. ydropisim. dat is de watersucht oder vor de kolde suke Terciana ghenomet. Man sieht an den beiden Beispielen auch, dass die beiden Elemente terminologisch gleichwertig sein können, die mnd. Formulierung kann aber auch eine weniger spezifische Erläuterung, z.B. durch einen Überbegriff, darstellen. Die Auswertung dieser Konstruktionen vor dem Hintergrund der ,Gart'-, ,Hortus'- und ,Gaerde'-Zusammenhänge ist ein Desiderat, das Aufschlüsse geben kann über den Stand der volkssprachigen Terminologie-Entwicklung und auch über das Verhältnis von mnd., hd. und lat. Terminologie.

Zum Bereich des Heilgeschehens gehört auch der Wortschatzsektor mit Bezeichnungen für die Art der Anwendung von zubereiteten Heilmitteln, dies sind typischerweise Verben wie bestreken 'bestreichen, äußerlich durch flächiges Bestreichen anwenden', dar up leghen 'auflegen', eten 'essen, innerlich einnehmen', smeren '(mit einer Flüssigkeit) einreiben', streken (up) ‘äußerlich auftragen', darin strouwen '(ein Pulver) wohinein streuen'. Seltener begegnen auch nominale Ausdrucksweisen für Arten der Anwendung wie z.B. roek 'Rauch' als Grundelement einer Räucherung.

vj II Eyn roek ghemaket van dissen bladen (vnde de vrowe van vnderup dar mede gherokert) reniget de moder. vnde sterket de borth. (Kap. 281) 
Ein dritter Wortschatzsektor bezieht sich auf die pharmakologische Praxis der Pflanzennutzung. Die im Gaerde behandelten Pflanzen werden als Drogen und damit als Gegenstände der pharmakologischen Praxis beschrieben, die eng auf den medizinischen Aspekt des Heilpotenzials bezogen ist. $\mathrm{Zu}$ den darauf bezogenen Teilwortschätzen gehören zunächst nicht wortgebildete Ausdrücke für arzneilich wirksame Pflanzenpräparate wie z.B. sap 'Saft', saet 'Same', kruet 'Pflanzenblätter' und Komposita wie z.B. wormden sap 'Wermutsaft'. Hierher gehören weiterhin auch Bezeichnungen für (pflanzenbasierte) Arzneiformen wie z.B. plaster, sirop oder electuarium, die, wenn sie fremdsprachiger Herkunft sind, jeweils unterschiedliche Grade der Integration aufweisen können. Zu electuarium begegnen zum Beispiel in unmittelbarer textueller Nachbarschaft sowohl die eingedeutschte Pluralform electuarien als auch die ursprüngliche lat. Form electuaria (Kap. 467). An früherer Stelle (Kap. 10) wird der Ausdruck explizit eingeführt: vnde make dar vth eyne menginghe (ghenomet electuarium).

Ein weiterer wichtiger lexikalischer Bereich sind die Bezeichnungen für Handlungsweisen bei der Beschreibung der Zubereitung von Heilmitteln. Diese Bezeichnungen sind mehrheitlich Verben, bei denen in vielen Fällen der Denkhintergrund der Säftelehre ebenfalls zu berücksichtigen ist, vor allem, wenn es um die Prinzipien der Mischung von Substanzen geht: togeven '(im Hinblick auf humorale Prinzipien) hinzugeben, hinzumischen, temperieren', menghen, vormengen 'durcheinander mischen', stoten 'in einem Mörser zerkleinern', tempereren 'mischen (und damit die humorale Natur mildernd beeinflussen)'.

Die Beispiele, die sich vermehren ließen, sollen zeigen, dass die übergeordnete Funktion von Pflanzenmonographien, ihre thematische Zerlegung und der medizinische Wissenshintergrund den Wortgebrauch und auch die Strukturierung des Wortschatzes tiefgreifend prägen.

\subsection{Wortgebrauch und Textfunktionen}

Neben den thematisch geprägten Teilwortschätzen gibt es andere, die auf bestimmte textuelle Funktionen bezogen sind. Sie sind mehrheitlich nicht spezifisch für medizinische Texte oder für Drogen-Monographien, sie sind aber dennoch wichtig für verschiedene kommunikative Aufgaben, die im Rahmen dieser Texte sprachlich realisiert werden müssen. Auch für diese Perspektive folgen wiederum drei Beispiele.

(i) Konnektoren sind Ausdrücke, mit denen Zusammenhänge zwischen Textteilen bzw. zwischen den damit vollzogenen sprachlichen Handlungen oder kommunikativen Aufgaben signalisiert werden können. Betrachten wir folgendes Beispiel aus dem Kap. 3 (die Konnektoren sind hervorgehoben): 


\begin{abstract}
पा Platearius sprikt. dat wormede eyne wedderwerdige nature an sik hebbe. w e n te se laxeret vnde stoppet. vnde de twe sint wedder eynander. Vnde d a r u m me sprikt he. dat wormede ghenuttet scal werden mit vormenginge. vnde dat angheseen werde ghebrek des minschen. We n te is id sake dat de nature des mynschen vorstoppet sy so scal men der wormde to geuen dinge de er de nature der stoppinghe benemen. vnde alleene laxere Is yd ou er $s$ a ke dat de nature to vele vletende weere. $s$ o scal men der wormde benemen de nature des vlotes. vnde to gheuen dinge de dar stoppen.
\end{abstract}

Mit wente und darumme wird jeweils signalisiert, dass der folgende Textteil eine Begründung darstellt für das vorher Behauptete. Eine vergleichbare Funktion erfüllt an anderer Stelle auch uppe dat 'auf dass, damit' (Vrouwen de kindere sogen. de scholen nicht nutten merk. $v$ p e d at se nicht vnsinnich werden [...]; Kap. 6); hier wird ein Verbot mit negativen Konsequenzen begründet, die bei Befolgung des Verbots vermieden werden. Die Wendung is id sake wird gebraucht, um eine Bedingung zu formulieren, in unserem Beispiel handelt es sich um zwei paarig formulierte alternative Bedingungen, die durch den Gebrauch von ouer 'aber' kontrastiert werden. Mit dem Gebrauch von so kann signalisiert werden, dass nun die Konsequenz zur vorher formulierten Bedingung folgt.

Diese wenigen Beispiele sollen zeigen, dass auch vordergründig ,harmlose‘ Ausdrücke wie die Konnektoren sehr eng mit der epistemischen Substanz des Textes verbunden sind. Wo in einem solchen Text Begründungen formuliert werden, geht es im Kern um systematische fachliche Zusammenhänge, die an der sprachlichen Oberfläche signalisiert werden.

(ii) Querverweisausdrücke können unter anderem genutzt werden, um zu signalisieren, dass Redegegenstände oben in einem Text bereits erwähnt wurden, oder um Hinweise auf später folgende Darstellungseinheiten zu geben. Im Mittelund Frühneuhochdeutschen dienen hierfür zunächst lexikalische Mittel, die ein gut ausgebautes Wortfeld bilden, hierzu gehören u.a. fest etablierte Ausdrücke wie obgemelt, ehegedacht oder mehr bemelt (vgl. Gloning 2003, Kap. 4). Im Gaerde sind hierzu u.a. folgende mnd. Gegenstücke zu belegen: berord, hyr na gherord, obgherord, upghenomed, upgherord, vorberord. Im Hinblick auf die Tradition dieses Wortschatzsektors muss man aber sagen, dass er im Gaerde zwar vorkommt, aber vergleichsweise schwach ausgebildet ist. $\mathrm{Zu}$ erklären ist dieser Befund wohl mit der vergleichsweise hohen Eigenständigkeit der einzelnen Pflanzenmonographien. Neben diesen lexikalisch realisierten Querverweisen finden sich selten auch textuelle Verfahren, wie zum Beispiel im Kap. 46, wo eine Pflanze mit einer anderen verglichen wird, auf deren Beschreibung dann verwiesen wird:

Aspergula Sternenkruet xlvi. cap.

ASpergula latinisch.

j Iा De mestere der arstedye spreken. dat Aspergula eyn luttick kruet sy vnde hefft wenich blade. vnde hefft welke knodighe stengele. vnde up eyneme iewelken knode hefft id blade 
vmmendumme. v. vj. effte vij. gheschicketgelik den sternen. als ok dat kruet hefft (Rubea ghenomet) dar van ghescreuen steit in deme boestaff $R .[\ldots]$

Funktional verwandt ist auch der Gebrauch von also bzw. alzo im Sinne von 'so wie beschrieben, in der eben beschriebenen Weise', der es erlaubt, sich zurückzubeziehen auf bereits beschriebene Heilanzeigen bzw. Zubereitungsformen in der unmittelbaren Textnachbarschaft. Im folgenden Beispiel aus Kap. 23 wird im Textteil viij die Zubereitung und die Anwendungsweise aus dem vorangehenden Textteil vij sozusagen inhaltlich herüberkopiert mit dem Gebrauch von also:

vij Ф Disse wortel ghelecht in wine mit dẽ krude nacht vnde dach (ghenomet paritaria) ghesaden vnde den ghedrunken. weeket den buck vnde benympt dat wee viij ๆा Ok is se (a ls o ghenuttet) gut vor fenynsche bete an deme lichamme (wor de syn moghen.)

(iii) Der Vergleich von Gegenständen bzw. die vergleichende Bewertung von Gegenständen, hier Pflanzen, ist eine weitere prominente textuelle Teilfunktion. Vergleiche werden unter anderem genutzt bei der Charakterisierung von Pflanzen, aber auch bei der vergleichenden Bewertung ihres Heilpotenzials. Eine der Konstruktionen für Vergleiche ist ein syntaktisches Muster, bei dem zwei Gegenstände (Pflanzen, Pflanzenteile) X und Y mit dem Verb gheliken verbunden werden und bei dem ggf. mit einem weiteren Textteil, der lexikalisch durch einen Konnektor eingeleitet wird, auf Unterschiede zwischen den Gegenständen hingewiesen werden kann.

$\mathrm{X}$ gheliket $\mathrm{Y}$, men dat $\mathrm{Y}<$ Angabe eines Unterschieds zu X>

Arthemisia sy eyn krut. $\mathrm{g}$ heli $\mathrm{k}$ et der wormede vnde der euerrude an der ghestalt. me $n$ d at de byoet breder blade hefft. (Kap. 1)

Solche Beispiele zeigen, dass auch das Zusammenspiel von syntaktischen Mustern und von lexikalischen Einheiten, die im Rahmen dieser Muster eingebaut werden können, eine lohnende Fragestellung ist.

Die vergleichende Beurteilung bzw. Bewertung von Heilmittelpflanzen bzw. Teilen oder Arten von Pflanzen ist eine der optionalen Informationspositionen in der pharmakologischen Texttradition. Im Gaerde findet sich hierfür u.a. ein syntaktisches Muster mit einer Komparativform eines Adjektivs:

$\mathrm{X}$ ist / synt beter / bequemer / nutter wen $\mathrm{Y}<$ im Hinblick auf ...>

xxiij Platearius sprikt. dat disses krudes blade b equ e mer synt in der arstedye. wen de wortele. vnde groen $n$ u t t er wen dorre. (Kap. 1) 
Das folgende Beispiel zeigt aber, dass einzelne Aspekte durchaus variabel im Textzusammenhang formuliert werden können, z.B. die relevanten Gegenstände der Bewertung, hier sind dies zwei Arten einer Pflanze und eine Reihe von Heilanzeigen:

ij पा De werdigen mestere der arstedye spreken eyndrechtliken. dat acetosa sy twyerleye. de eyne groet. de andere klene. De grote hefft lange stengele vnde bouen dar an knobbeken glik der groten klyuen. vnde de is ghenomet Acetosa maior. De lutticke hefft klene blade. de synt vet. vnde hefft eynen dunnen stengel. vnde klene saet. vnde disse is ghenomet accedula effte Acetosella. vnde is ok droger vnde kolder nature iij $\|$ Vnde de $n$ et der heten magen. vnde to der quaden leueren. vnde to dem herten. $v$ nde bringet lust to etende. Men to dissen allen is de grote Ac e to s a beter. (Kap.13)

Ich breche die Besprechung des Zusammenhangs von Wortgebrauch und textfunktionalen Aufgaben hier ab. Die Beispiele sollten zeigen, dass der Wortgebrauch des Gaerde auch von den mehr oder weniger spezifischen textfunktionalen Aufgaben geprägt ist, die in einer Pflanzenmonographie vorkommen können. Weitere funktionale Kategorien dieser Art sind z.B. Mengenangaben (eyne hant vul; eyn quentyn; eyn halfloet), Formen der Charakterisierung des äußeren Erscheinungsbildes einer Pflanze (z.B. mit Adjektiven wie ghedelet in Bezug auf eine Blüte) oder die Formulierung von Bezeichnungs- bzw. Erläuterungszusammenhängen (mit Gelenkausdrücken wie dat is oder ghenomet). Auch die nicht seltenen Doppel- oder Paarformeln (nympt wech vnde vordrifft) müssen genauer untersucht werden, da hier ganz unterschiedliche semantische und funktionale Zusammenhänge zwischen den Elementen bestehen können.

\subsection{Die Rolle der Wortbildung}

Wie viele andere Fachtexte ist auch der Gaerde reich an Wortbildungen, die in der Regel eng mit der funktionalen und der thematischen Prägung des Textes zusammenhängen. Ich möchte auch diese Zusammenhänge in exemplarischer Weise veranschaulichen, indem ich ausgewählte Wortbildungsphänomene in eine kommunikative Perspektive stelle.

(i) Eine wichtige Funktion im Rahmen von Pflanzenmonographien ist es, Teile von Pflanzen im weitesten Sinne anzusprechen. Hierfür dienen Determinativkomposita, in denen Ausdrücke wie sap, wortel, blome, kern usw. als Determinatum kombiniert werden mit einer Pflanzenbezeichnung als Determinans, z.B. wormden sap, porrys sap, leckerissen sap; dusentgulden blomen, olyuen blomen, borrich blomen, camellen blomen / camellenblomen, muscatenblomen; persik kerne, mandelkerne, oliven kerne, quedenkerne / queden kerne. Solche Komposita 
werden mal zusammengeschrieben, mal nicht, zum Teil findet man in direkter textueller Nachbarschaft beide Varianten. Auch unter den Pflanzenbezeichnungen selbst finden sich viele Wortbildungen wie z.B. hertestunge 'Hirschzunge', holwoert oder sternekruet.

(ii) Im Bereich der Krankheitsbezeichnungen konkurrieren Wortbildungen mit syntaktischen Strukturen. So findet man einerseits Wortbildungen wie bloetgang oder darmsuke, lungen suke und swynt suke, andererseits nominale Strukturen mit Genitivattributen wie vloete des buekes oder eine lange Reihe von Bezeichnungen mit wee als Kern: wee der teenen, wee des bukes, wee der blazen, wee der lenden, wee des ghedermtes, wee des houedes, wee der oren, wee der borst, wee der lungen, wee des stertes, wee der huffte, wee der moder. Im Text werden solche Wortbildungen und Wendungen vor allem bei der Charakterisierung der Heilanzeigen gebraucht:

ij Ф Paulus in dẽ capi. Fraxinus sprikt: dat dysse boem sy kolt vnde droge an dẽ anderen grade. Vnde sprikt dat de borke disses bomes in regenwater ghesaden (vnde dar van ghenuttet) is guet vor den vlote des buekes vndevor de derme suke.vnde okvor den bl o e t g a n c k. (Kap. 216; vgl. de dermsuke Colica ghenomet, Kap. 228)

(iii) Es gibt zahlreiche weitere Beispiele, wie Wortbildungen einzelnen Teilfunktionen zuzuordnen sind, so z.B. hittig zur Charakterisierung einer Primärqualität, suntmaken zur Charakterisierung einer Sekundärqualität, waterlaten zur Bezeichnung einer Körperfunktion, teenenvlesch zur Bezeichnung eines Körperteils oder auch Ableitungen zur Bezeichnung der primären humoralen Dimensionen:

Cera was cxlii. cap.

CEra latinsch. Vamacha arabisch.j II Serapio sprikt / dat was to velen dingen guet sy (uthwendich des lichammes vnde nicht inwendich) vnde is in dẽ middele tempereret mit den ve er qualite te n. als an derwermde. an derkolde. an dervuchticheit.vnde ander drogicheit

Umgekehrt kann man zeigen, dass auch einzelne Wortbildungsverfahren ein spezifisches Funktionsprofil im Rahmen eines solchen Textes haben können. So findet man zahlreiche Wortbildungen auf -(h)eit: bequemicheit, boszheit, drogicheit, drunchenheit, dusterheit (der ogen), geylicheit, hardicheit, koldicheit, krankheit, lamicheit, rodicheit, scharpheit, serichheit, soticheit, strengicheit, swarticheit, unkuscheit, utsettischeit, veleheit, vetticheit (effte olye), vuchticheit. Diese Ausdrücke beziehen sich mehrheitlich auf Eigenschaften im weitesten Sinne, die bei der medizinisch-botanischen Charakterisierung angesprochen werden, teilweise auch auf Gegenstände (Krankheiten, Substanzen).

(iv) Manche Wortbildungen sind Gegenstücke zu fremdsprachigen Ausdrücken, das erkennt man besonders klar in Paarformeln bzw. in Erklärungskonstel- 
lationen, z.B. in folgender Heilanzeige, aus der hervorgeht, dass die Wortbildung dyalten salue das volkssprachige Gegenstück zur genitivischen Wendung unguentum dyalteae ist:

Vnde vthwendich scal men smeren de borst mit bottere. effte mit eyner saluen. ghenomet (vngentum dyaltee) dat is dyalten salue. (Kap. 2)

Aber auch eine Ableitung wie sterkinghe hat in lateinisch confortativum ein terminologisches Gegenstück, ${ }^{10}$ was in einer der Textpassagen auch explizit erläutert wird:

Dar na is guet to nuttende eyne sterkinghe. ghenomet dyapenidion. effte diayris salomonis. (Kap. 2); vnde dar na scal men bruken een sterkinge ghenomet dyalacca. (Kap. 22); To disser krankheyt / voerghescreuen. is guet eyne sterkinge ghenomet Confectio anacardina. (Kap. 33); vnde dar na ghenuttet eyne sterkinge ghenomet Triasandali (Kap. 105); $v$-ा Van kerseberen maket men eyn Confortatiuum (dat is) eine sterkinge ghenomet dyaceraseon. (Kap. 130)

Es gehört zu den zukünftigen Aufgaben, die Grade der Etabliertheit der unterschiedlichen Wortbildungen und ihrer fachlichen Verwendungsweisen textgestützt und unter Berücksichtigung der Wörterbuchbefunde zu bestimmen. Die Rolle der Wortbildung muss auch berücksichtigt werden, wenn die terminologischen Zusammenhänge zwischen den mittel- und frühneuhochdeutschen, den mnd. und den lat. Wörtern bzw. Verwendungsweisen und die Zusammenhänge in den fachlichen Wortschatzarchitekturen, die sich ja alle mehr oder weniger auf dasselbe medizinische Denksystem beziehen, bestimmt und textgestützt dokumentiert werden.

\subsection{Lexikalische Mittel fremdsprachiger Herkunft}

Im Gaerde ist auch ein Bestand lexikalischer Mittel fremdsprachiger Herkunft zu verzeichnen, der sich in Grundzügen folgendermaßen charakterisieren lässt. Zum einen finden wir zitiertes fremdsprachiges Material, unter anderem in den Textteilen zu den arab., griech. usw. Pflanzenbezeichnungen, zum anderen lassen sich vor allem lat. Wörter und Wendungen aus der medizinischen Terminologie belegen, die zum Teil auch als solche gekennzeichnet sind (to latine flecma) bzw. in den genannten Erläuterungskontexten mit dat is oder ghenomet vorkommen

10 Bei Schiller / Lübben nicht gebucht. Im MndHwb III, 472 mit einer Belegstelle aus dem Gaerde. 
(de dulheit des houedes frenesis ghenomet; secundinam. dat is de ander bort; dat saet gehenomet sperma).

Sodann gibt es aber auch einen breiten Wortschatzbereich, dessen Elemente mehr oder weniger deutliche Zeichen der Entlehnung aufweisen, die teilweise auch durch die Wortgeschichte gestützt werden, z.B. Wörter wie tempereren, laxeren, pulveren, complexie, substancie, pestilencie, subtyl, fantasie, memorie. Oben wurde bei den Bezeichnungen für Arzneiformen schon darauf hingewiesen, dass es Wörter fremdsprachiger Herkunft gibt, die zum Teil in unmittelbarer Nachbarschaft eingedeutschte und lat. Formen aufweisen (electuarien, electuaria). Wörter wie dyamargariton und andere Bezeichnungen für pharmakologische Kompositionen orientieren sich mehrheitlich an den griechischen Formen, die so allerdings auch in der lat. Fachliteratur (z.B. Saladin von Asculo; Luminare maius) verwendet werden.

Viele dieser mnd. Wörter fremdsprachiger Herkunft haben Gegenstücke im Mhd. und Fnhd., z.B. laxieren, temperieren, complexion und viele andere. Die Parallelität betrifft nicht nur die Wörter, sondern auch die zum Teil spezifischen Verwendungsweisen der Vier-Säfte-Lehre und der Medizin im weiteren Sinne, z.B. nature als Bezeichnung für die gesamte Schöpfung (de wunderlike werke des scheppers der nature), als Bezeichnung für die humorale Prägung von Lebewesen und Substanzen (de veer nature der element. als hitte. kolde. vuchticheit vnde drogeheit), aber auch als Bezeichnung für den männlichen Samen, das Sperma (dat saet ghenomet sperma. dat is de menlike nature; dẽ de nature entgeyt ane synen willen).

Ich muss es bei diesen ersten Bemerkungen bewenden lassen und komme zu einer Zusammenfassung mit einem Ausblick.

\section{Ergebnisse und Aufgaben}

Im vorliegenden Beitrag habe ich in exemplarischer Weise erste Befunde zu Fragen der Textorganisation und zum Wortgebrauch im mnd. Gaerde der suntheit (1492) dargelegt.

Im Mittelpunkt standen zum einen Aspekte der textuellen Schematisierung von Pflanzenmonographien, die den wesentlichen Teil dieses Werkes ausmachen, zum anderen die Frage nach der typischen Organisation und den Realisierungsspielräumen zentraler funktionaler Textbausteine wie der Heilanzeige. Dabei hat sich gezeigt, dass sich die Textorganisation weitgehend im Rahmen der üblichen textuellen Verfahrens- und Schematisierungsweisen der pflanzenkundlichen und pharmakologisch-medizinischen Literatur des Mittelalters und der Frühen Neuzeit bewegt. 
Im Hinblick auf den Wortgebrauch und den Wortschatz wurde zunächst gezeigt, wie sich einzelne Wortschatzsektoren zum einen entlang von thematischen und fachlichen Strukturaspekten des Textes (z.B. Krankheitsbezeichnungen, Grundlagen der Vier-Säfte-Lehre), zum anderen entlang von textfunktionalen Gesichtspunkten (z.B. sprachliche Mittel der Begründung, des Querverweises, der Quellenkennzeichnung) beschreiben lassen. In weiteren Abschnitten wurden die Rolle der Wortbildung und der Wortbestand fremdsprachiger Herkunft in Umrissen charakterisiert.

Dabei wurde auch deutlich, wie hilfreich eine digitale Volltext-Transkription des Gaerde-Textes ist, die bereits in den 1990er Jahren von Christel und Peter Seidensticker erarbeitet wurde und die jüngst von einer Arbeitsgruppe im CLARIND-Projekt nach modernen TEI-konformen Standards für das Deutsche Textarchiv aufbereitet wurde.

$\mathrm{Zu}$ den zukünftigen Aufgaben gehören unter anderem folgende Schritte:

- Vergleich des Textbestandes und der sprachlichen Gestalt des Gaerde mit den Paralleltexten im hochdeutschen Gart (1485) und im lateinischen Hortus (1491);

- umfassende und detaillierte Dokumentation der Wortgebrauchs- und Wortschatzzusammenhänge im mnd. Gaerde, im fnhd. Gart und im lateinischen Hortus, ggf. auch der Texte aus dem relevanten Quellenraum;

- detaillierter Abgleich der im Gaerde genutzten textuellen Verfahren und thematischen Praktiken mit den Texten aus der älteren medizinisch-pharmakologischen Literatur in hoch- und mittelniederdeutscher sowie in lateinischer Sprache;

- Einordnung der sprachlichen Befunde in die bisherigen Forschungen zur mnd. Sprach- und Fachprosageschichte ${ }^{11}$ sowie

- weitere Korrektur und Verbesserung der vorhandenen digitalen Arbeitstranskription, ggf. auch weitere computerlinguistische Aufbereitung im Umkreis der Arbeiten am mittelniederdeutschen Referenzkorpus.

11 Bezugspunkte bieten u.a. die Überblicke im HSK-Werk zur Sprachgeschichte von Möhn / Schröder (2000), Cordes / Niebaum (2000) und Meier / Möhn (2000). 


\section{Literatur}

\subsection{Quellen}

Anholter-Moyländer Kräuterbuch. Das Kräuterbuch von Johannes Hartlieb in einer um 1470 entstandenen Abschrift aus der Fürstlich Salm-Salm'schen Bibliothek der Wasserburg Anholt FSSB Ms. 46, Faksimile-Ausgabe, Stiftung Museum Schloß Moyland (Hrsg.) (2004), Bedburg-Hau.

Das „Speyerer Kräuterbuch“ mit den Heilpflanzen Hildegards von Bingen. Eine Studie zur mittelhochdeutschen „Physica“-Rezeption mit kritischer Ausgabe des Textes, Barbara Fehringer (Hrsg.) (1994), (Würzburger medizinhistorische Forschungen, Beiheft 2), Würzburg.

Gaerde der suntheit - Hiir heuet an de lustighe vnde nochlighe Gaerde der suntheit, [Exemplar der SUB Göttingen] (1492), Lübeck.

Gart der gesuntheit, Peter Schöffer (1485), Mainz. Nachdruck München-Allach: Kölbl (1966). Hortus sanitatis - Ortus sanitatis, [Exemplar der BSB München] (1491), Mainz.

Ortus sanitatis. De herbis et plantis. De Animalibus et reptilibus. De Auibus et volatilibus. De Piscibus et natatilibus. De Lapidibus (...) Tabula medicinalis cum directorio generali per omnes tractatus, (1511), Venedig. Nachdruck in zwei Bänden. Popp (1978), Würzburg.

Der deutsche „Macer“(Vulgatfassung). Mit einem Abdruck des lateinischen Macer Floridus „De viribus herbarum“, Bernhard Schnell / William Crossgrove (Hrsg.) (2003), Niemeyer, Tübingen.

Das Promptuarium medicinae. Magdeburg, Bartholomäus Ghotan (1483), Peter Seidensticker (Hrsg.) (1990), (Corpus herbariorum, 1), Lahr.

\subsection{Sekundärliteratur}

Anholter-Moyländer Kräuterbuch = Müller, Irmgard / Martin, Michael / Wiehl, Peter (Hrsg.) (2004), Anholter-Moyländer Kräuterbuch, Wissenschaftlicher Begleitband zur FaksimileAusgabe, Bedburg-Hau.

Baumann, Susanne (1998), Pflanzenabbildungen in alten Kräuterbüchern. Die Umbelliferen in der Herbarien- und Kräuterbuchliteratur der frühen Neuzeit (Heidelberger Schriften zur Pharmazie- und Naturwissenschaftsgeschichte, 15), Stuttgart.

Cordes, Gerhard / Hermann Niebaum (2000), „Wortbildung des Mittelniederdeutschen“, in: Werner Besch u.a. (Hrsg.), Sprachgeschichte. Ein Handbuch zur Geschichte der deutschen Sprache und ihrer Erforschung, Teilbd. (HSK, 2, 2), 2., vollst. neu bearb. und erw. Aufl., Berlin / New York, 1463-1469.

Fritz, Gerd (2013), Dynamische Texttheorie. Gießen: Gießener Elektronische Bibliothek. URL: http://geb.uni-giessen.de/geb/volltexte/2013/9243

Gerhardt, Christoph / Bernhard Schnell (2002), In verbis in herbis et in lapidibus est deus. Zum Naturverständnis in den deutschsprachigen illustrierten Kräuterbüchern des Mittelalters, Trier.

Gloning, Thomas (2007), „Deutsche Kräuterbücher des 12. bis 18. Jahrhunderts. Textorganisation, Wortgebrauch, funktionale Syntax“, in: Andreas Meyer / Jürgen Schulz-Grobert 
(Hrsg.), Gesund und krank im Mittelalter. Marburger Beiträge zur Kulturgeschichte der Medizin. 3. Tagung der Arbeitsgruppe „Marburger Mittelalter-Zentrum“, Marburg, 25. und 26. März 2005, Leipzig, 9-88.

Gloning, Thomas (2010), „Funktionale Textbausteine in der historischen Textlinguistik. Eine Schnittstelle zwischen der Handlungsstruktur und der syntaktischen Organisation von Texten “, in: Arne Ziegler (Hrsg.), Historische Textgrammatik und Historische Syntax des Deutschen. Traditionen, Innovationen, Perspektiven, Bd. 1: Diachronie, Althochdeutsch, Mittelhochdeutsch, Berlin / New York, 173-193.

Gloning, Thomas (2011a), „Humoraler Wortgebrauch in der Prosavorrede zum deutschen ,Macer“ (13. Jh.)“, in: Ralf Plate / Martin Schubert (Hrsg.), Mittelhochdeutsch. Beiträge zur Überlieferung, Sprache und Literatur. Festschrift für Kurt Gärtner zum 75. Geburtstag, Berlin / Boston, 375-386.

Gloning, Thomas (2011b), „Spielarten der Quellenkennzeichnung in Fachtexten des Mittelalters und der Frühen Neuzeit“, in: Mechthild Habermann (Hrsg.), Textsortentypologien und Textallianzen des 13. und 14. Jahrhunderts (Berliner sprachwissenschaftliche Studien, 22), Berlin, 303-332.

Habermann, Mechthild (2002a), Deutsche Fachtexte der frühen Neuzeit. Naturkundlich-medizinische Wissensvermittlung im Spannungsfeld von Latein und Volkssprache (SLG, 61), Berlin / New York.

Habermann, Mechthild (2002b), „Kräuterbücher im Wandel. Untersuchungen zur Textorganisation an ausgewählten Werken des 15. bis 18. Jahrhunderts “, in: Franz Simmler (Hrsg.), Textsorten deutscher Prosa vom 12. / 13. bis 18. Jahrhundert und ihre Merkmale. Akten zum Internationalen Kongress in Berlin, 20. bis 22. September 1999 (JIG, 67), Bern u.a., 552-571.

Habermann, Mechthild (2003), „Der Sprachenwechsel und seine Folgen. Zur Wissensvermittlung in lateinischen und deutschen Kräuterbüchern“, in: Sprachw., 28, 325-354.

Harms, Heinz (1968), „Die pflanzlichen Arzneistoffe einer mittelniederdeutschen hippiatrischen Rezeptsammlung des 16. Jahrhunderts“, in: Gundolf Keil u.a. (Hrsg.), Fachliteratur des Mittelalters. Festschrift für Gerhard Eis, Stuttgart, 293-306.

Hayer, Gerold (Hrsg.) (1997), Konrad von Megenberg, ,Das Buch der Natur: Johannes Hartlieb, ,Kräuterbuch؛ Farbmikrofiche-Edition der Handschrift Heidelberg, Universitätsbibliothek, Cod. Pal. Germ. 311 und der Bilder aus Cod. Pal. germ. 300 (Codices illuminati medii aevi, 33), München.

Hayer, Gerold (1998), Konrad von Megenberg ,Das Buch der Natur: Untersuchungen zu seiner Text- und Überlieferungsgeschichte (MTU, 110), Tübingen.

Hoppe, Brigitte (1969), Das Kräuterbuch des Hieronymus Bock. Wissenschaftshistorische Untersuchung mit einem Verzeichnis sämtlicher Pflanzen des Werkes, der literarischen Quellen der Heilanzeigen und der Anwendungen der Pflanzen, Stuttgart.

Jellinghaus, Hermann (1925), Geschichte der mittelniederdeutschen Literatur (Grundr., 7), 3., verb. Aufl., Berlin / Leipzig.

Keil, Gundolf (1980), „Gart der Gesundheit“, in: Kurt Ruh u.a. (Hrsg.), VL, Bd. 2: Comitis, Gerhard Gerstenberg, Wigand. Com - Ger, 2., völlig neu bearb. Aufl., Berlin / New York, 1072-1092.

Keil, Gundolf (1983), „Hortus sanitatis“, in: Kurt Ruh u.a. (Hrsg.), VL, Bd. 4: Hildegard von Hürnheim - Koburger, Heinrich. Hil - Kob, 2., völlig neu bearb. Aufl., Berlin / New York, 154-164.

Keil, Gundolf (1993), „Ortolf-Anteile im ,Promptuarium medicinae‘. Untersuchungen zur Textschleppe von Bartholomäus Ghotans mittelniederdeutschem Kräuterbuch“, in: 
Gundolf Keil (Hrsg.), „ein teutsch puech machen“. Untersuchungen zur landessprachlichen Vermittlung medizinischen Wissens (Ortolf-Studien, 1), Wiesbaden, 499-537.

Keil, Gundolf (2002), „Standardwerke mittelalterlicher Drogenkunde und ihre Repräsentation in der Enzyklopädik“, in: Christel Meier (Hrsg.), Die Enzyklopädie im Wandel vom Hochmittelalter bis zur frühen Neuzeit (MM-S, 78), München, 343-389.

Keil, Gundolf / Peter Dilg (2003), „Kräuterbücher“, in: LexMA, Bd. 5: Hiera-Mittel - Lukanien, München, 1476-1480.

Keil, Gundolf (1982), „,Gart‘, ,Herbarius‘, ,Hortus‘. Anmerkungen zu den ältesten KräuterbuchInkunabeln“, in: Gundolf Keil (Hrsg.), „gelêrter der arzenîe, ouch apotêker“. Festschrift Willem F. Daems, Pattensen, 589-635.

Lindgren, Agi (Hrsg.) (1967), Ein Stockholmer mittelniederdeutsches Arzneibuch aus der zweiten Hälfte des 15. Jahrhunderts (AUS, 5), Stockholm / Göteborg / Uppsala.

Lübben, August (1888 / 1990), Mittelniederdeutsches Handwörterbuch, nach dem Tode des Verfassers vollendet von Christoph Walther, Leipzig, Nachdruck Darmstadt 1990.

Mayer, Johannes Gottfried (2000), „Das ,Leipziger Drogenkompendium“ (Leipzig, UB, Cod. 1224) und seine Quellen“, in: Johannes Gottfried Mayer / Konrad Goehl (Hrsg.), Editionen und Studien zur lateinischen und deutschen Fachprosa des Mittelalters. Festschrift Gundolf Keil, Würzburg, 207-263.

Mayer, Johannes Gottfried / Konrad Goehl (2003), „Das Verhältnis der mittelalterlichen deutschen Kräuterbücher zu ihren lateinischen Quellen, dargestellt am ,Älteren deutschen Macer', dem ,Leipziger Drogenkompendium‘ (Leipzig UB, 1224) sowie dem ,Gart der Gesundheit‘ (Mainz 1485)“, in: Sprachw., 28, 273-292.

Mayer, Johannes G. (1995), „, Circa instans“ deutsch. Beobachtungen zum Leipziger Kodex 1224, dem bislang umfangreichsten Kräuterbuch in deutscher Sprache vor dem Buchdruck“, in: Würzburger medizinhistorische Mitteilungen, 13, 67-73.

Meier, Jürgen / Dieter Möhn (2000), „Die Textsorten des Mittelniederdeutschen“, in: Werner Besch u.a. (Hrsg.), Sprachgeschichte. Ein Handbuch zur Geschichte der deutschen Sprache und ihrer Erforschung, Teilbd. (HSK, 2, 2), 2., vollst. neu bearb. und erw. Aufl., Berlin / New York, 1470-1477.

Meier-Staubach, Christel (1996), „,Der ,Hortus sanitatis‘ als enzyklopädisches Buch. Zur Pragmatisierung traditionellen Wissens und ihrer Realisierung in der Illustration“, in: Hans Höfinghoff u.a. (Hrsg.), Alles was Recht war. Festschrift Ruth Schmidt-Wiegand, Essen, 191-200.

Mittelniederdeutsches Handwörterbuch (1928ff.), begründet von Agathe Lasch / Conrad Borchling. Fortgef. von Gerhard Cordes. Ab Lfg. 24 hrsg. von Dieter Möhn, Bd. 1ff., Neumünster / Kiel / Hamburg.

Möhn, Dieter / Ingrid Schröder (2000), „Lexikologie und Lexikographie des Mittelniederdeutschen“, in: Werner Besch u.a. (Hrsg.), Sprachgeschichte. Ein Handbuch zur Geschichte der deutschen Sprache und ihrer Erforschung, Teilbd. (HSK, 2, 2), 2., vollst. neu bearb. und erw. Aufl., Berlin / New York, 1435-1456.

Müller, Irmgard / Michael Martin (2005), „Krankheitsbezeichnungen und Heilmittelgebrauch in einem neuen Überlieferungszeugen von Johann Hartliebs Kräuterbuch“, in: Das Mittelalter, 10 / 1, 132-144.

Norrbom, Sven (1921), Das Gothaer mittelniederdeutsche Arzneibuch und seine Sippe (Mittelniederdeutsche Arzneibücher, 1), Hamburg.

Payne, Joseph Frank (1903), „On the ,Herbarius“ and ,Hortus sanitatis““, in: Transactions of the Bibliographical Society, 6, 63-126.

Riecke, Jörg (2004), Die Frühgeschichte der mittelalterlichen medizinischen Fachsprache im Deutschen, 2 Bde., Bd. 1: Untersuchungen, Bd. 2: Wörterbuch, Berlin / New York. 
Riethe, Peter (2005), „Hildegards von Bingen ,Liber simplicis medicinae“ im Mainzer ,Gart der Gesundheit'“, in: Sudhoffs Archiv, 89 / 1, 96-119.

Sanders, Willy (1982), Sachsensprache, Hansesprache, Plattdeutsch. Sprachgeschichtliche Grundzüge des Niederdeutschen, Göttingen.

Schiller, Karl / August Lübben (1875-1881), Mittelniederdeutsches Wörterbuch, 6 Bde., Bremen.

Schnell, Bernhard (1991), „Das ,Prüller Kräuterbuch‘. Zum ersten Herbar in deutscher Sprache“, in: $Z d A, 120,184-202$.

Schnell, Bernhard (1999), „,Von den eynveldighen arcztyen‘. Zu den Quellen des Kräuterbuchs Leipzig Ms. 1224. Eine bislang unbekannte deutschsprachige Übersetzung des Ps.-Serapion“, in: Rudolf Bentzinger / Ulrich-Dieter Oppitz (Hrsg.), Fata libellorum (GAG, 648), Göppingen, 293-312.

Schnell, Bernhard (2000), „Gewürze in mittelalterlichen Kräuter- und Kochbüchern“, in: „Wo der Pfeffer wächst“. Ein Festival der Kräuter und Gewürze (Palmengarten, Sonderheft, 32), Frankfurt am Main, 81-90.

Schnell, Bernhard (2003), „Pflanzen in Bild und Text. Zum Naturverständnis in den deutschsprachigen illustrierten Kräuterbüchern des Spätmittelalters“, in: Peter Dilg (Hrsg.), Natur im Mittelalter. Konzeptionen - Erfahrungen - Wirkungen, Berlin, 442-561.

Seidensticker, Peter (1990). „Die frühen deutschen Kräuterbücher als Quelle für die Lexikographie“, in: Rudolf Schützeichel / Peter Seidensticker (Hrsg.), Wörter und Namen. Aktuelle Lexikographie (Symposium 1987), Marburg, 130-138.

Seidensticker, Peter (1993), „Was müssen das für Bäume sein... Tradition und Fabel in den Kräuterbüchern“, in: Ingrid Kühn / Gotthard Lerchner (Hrsg.), Von wyßheit würt der mensch geert ... Festschrift für Manfred Lemmer, Frankfurt am Main u.a., 241-255.

Seidensticker, Peter (1996), „Nomenklaturen: Pflanzennamen“, in: Ernst Eichler u.a. (Hrsg.), Namenforschung. Ein internationales Handbuch zur Onomastik, Teilbd. (HSK, 11, 2), Berlin / New York, 1622-1626.

Seidensticker, Peter (1997), „die seltzamen namen all“. Studien zur Überlieferung der Pflanzennamen. Grundzüge einer historischen Syntax der Pflanzennamen (ZDL, Beiheft 101), Stuttgart.

Seidensticker, Peter (1999), Pflanzennamen. Überlieferung, Forschungsprobleme, Studien (ZDL, Beiheft 102), Stuttgart.

Seidensticker, Peter (1999), „Botanik und Fachsprache in den Kräuterbüchern der Renaissance“, in: Lothar Hoffmann u.a. (Hrsg.), Fachsprachen. Ein internationales Handbuch zur Fachsprachenforschung und Terminologiewissenschaft (HSK, 14), Berlin / New York, 2401-2410.

Seidensticker, Peter (2001), „Kräuterbücher und Sprachwissenschaft. Ein Forschungsbericht“, in: Angelika Braun (Hrsg.), Beiträge zu Linguistik und Phonetik. Festschrift für Joachim Göschel zum 70. Geburtstag, Stuttgart, 80-94.

Seidensticker, Peter (2010), Aisthesis. Wahrnehmung der Farben in den Pflanzenbeschreibungen der frühen deutschen Kräuterbücher (ZDL, Beiheft 139), Stuttgart.

Temmen, Mareike (1998), Die medizinische Rezepthandschrift Burgsteinfurt Hs. 15. Edition und Untersuchung einer Handschrift aus dem 16. Jahrhundert (Westfälische Beiträge zur niederdeutschen Philologie, 7), Bielefeld.

Temmen, Mareike (2006), Das ,Abdinghofer Arzneibuch؛ Edition und Untersuchung einer Handschrift mittelniederdeutscher Fachprosa, Köln / Weimar / Wien.

Windler, Ernst (Hrsg.) (1932), Das Bremer mittelniederdeutsche Arzneibuch des Arnoldus Doneldey, mit Einleitung und Glossar, Neumünster. 